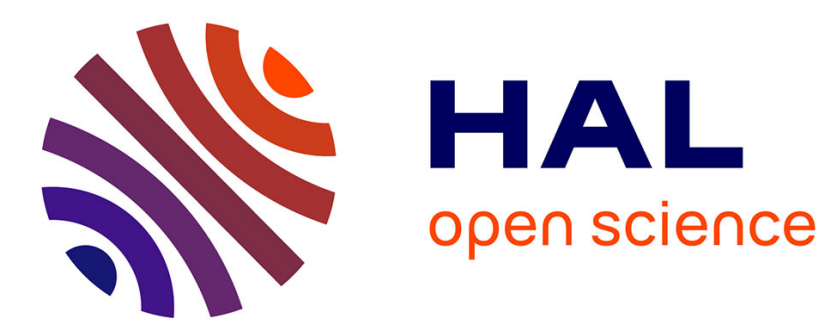

\title{
Sport et socialisation professionnelle. Le cas d'un raid organisé par une entreprise du CAC 40
}

Denis Bernardeau Moreau, Marie Hingant

\section{To cite this version:}

Denis Bernardeau Moreau, Marie Hingant. Sport et socialisation professionnelle. Le cas d'un raid organisé par une entreprise du CAC 40. Sociologies pratiques, 2016, 32, pp.33 - 33. 10.3917/sopr.032.0033 . hal-01395182

\section{HAL Id: hal-01395182 \\ https://hal.science/hal-01395182}

Submitted on 14 Nov 2016

HAL is a multi-disciplinary open access archive for the deposit and dissemination of scientific research documents, whether they are published or not. The documents may come from teaching and research institutions in France or abroad, or from public or private research centers.
L'archive ouverte pluridisciplinaire HAL, est destinée au dépôt et à la diffusion de documents scientifiques de niveau recherche, publiés ou non, émanant des établissements d'enseignement et de recherche français ou étrangers, des laboratoires publics ou privés. 


\title{
Sport et socialisation professionnelle Le cas d'un raid organisé par une entreprise du CAC 40
}

\author{
Denis BERNARDEAU-MOREAU ${ }^{1}$ et Marie HINGANT ${ }^{2}$
}

Résumé : En nous appuyant sur une trentaine d'entretiens menés auprès des salariés d'une grande entreprise du CAC 40, notre intention est de réfléchir sur les formes de socialisation professionnelle qu'induit un événement sportif interne de grande ampleur. Le fait de participer à l'une des éditions du Raid organisé par leur employeur renforce-t-il l'appropriation des valeurs de l'entreprise par les salariés ? Ces derniers portent-ils un regard différent sur leur entreprise et sa hiérarchie ? La socialisation particulière vécue durant les épreuves sportives conduit-elle les participants à modifier les relations professionnelles avec leurs collègues de travail ? Le sport peut-il être considéré comme un outil efficace de cohésion interne?

\section{MOTS-CLÉS : ÉVÉNEMENT SPORTIF - SOCIALISATION PROFESSIONNELLE - COMMUNICATION INTERNE - VALEURS DE L'ENTREPRISE - ATTACHEMENT ÉMOTIONNEL}

De nombreux travaux traitent des liens entre le monde de l'entreprise et celui du sport. Ces liens, soulignent Pierre et Barth $(2010$, p. 2) sont séculaires et pluriels. Le rôle fédérateur du sport, les avantages sociaux qu'il procure, la fonction de formation qu'il autorise, la communication institutionnelle qu'il permet de façonner, ses bienfaits sur la santé physique et mentale des salariés, son rôle d' " amortisseur social " sont soulignés par plusieurs auteurs (Ehrenberg, 1991 ; Meignant et Rayer, 1991 ; Sekiou et al., 1993 ; Barbusse, 1997 ; Fourré, 2000 et 2003 ; Burlot, 2001 ; Pierre, 2006 ; Lefebvre, 2007 ; Bertrand, 2008 ; Pierre et Barth, 2010 ; Pierre Pichot et Burlot, 2010). Le professionnalisme du sport des années 1980, souligne Barbusse (2009, p. 11), a contribué à rapprocher plus encore les organisations sportives et les logiques mana- gériales de l'entreprise, au risque notent Le Goff (1992), Barbusse (2002) ou encore Pierre et al. (2010), de favoriser une certaine instrumentalisation du sport ${ }^{3}$. Dans les associations dont la perméabilité aux enjeux de performance économique est crois- sante, on observe également une évolution professionnelle du profil des bénévoles (Loirand, 2003 ; Bernardeau, 2004). Entre sport et entreprise, les intérêts sont donc multiples et réciproques. "Les échanges entre le sport et l'entreprise ne se font plus à sens unique. L'entremêlement des deux mondes est devenu une réalité ", écrit Barbusse (2009, p. 19). Les valeurs du sport servent la politique managériale de

\footnotetext{
${ }^{1}$ Maître de conférences, laboratoire Analyse comparée des pouvoirs (ACP), Université Paris-Est Marnela-Vallée.

${ }^{2}$ Doctorante, ACP-UPEM.

${ }^{3}$ Cette instrumentalisation, note Barbusse, répond à une volonté première d'optimiser coûte que coûte la performance économique de l'entreprise (2002, p. 410).
} 
L'entreprise et sa culture mais en retour les dirigeants sportifs utilisent les méthodes entrepreneuriales pour optimiser leurs événements et leurs fonctionnements. Si tous ces travaux sont utiles pour attester ce " mariage de raison " et les liens étroits qui unissent désormais les entreprises à l'univers du sport, il est un sujet qui mérite d'être davantage approfondi. Le sport sert aussi à renforcer la socialisation professionnelle des salariés dans leur entreprise. Selon Dubar (2000), la socialisation professionnelle représente les processus formels et informels en vertu desquels un individu acquiert et développe les traits culturels et sociaux typiques d'un groupe professionnel. La question de savoir si le sport contribue à affermir l'identité professionnelle des personnels et à accroître leur attachement aux valeurs de l'entreprise, mérite d'être posée ${ }^{4}$. Par son action particulière, le sport permet-il réellement de densifier les rap- ports sociaux entre les salariés et leur entreprise?

Notre intention dans cet article est de prolonger cette réflexion en nous appuyant sur une trentaine d'entretiens menés auprès de salariés d'une grande entreprise du CAC $40^{5}$ ayant participé à l'une des éditions du Raid organisé par leur employeur. Parmi les personnes interviewées, on compte un tiers de cadre et deux tiers d'employés. L'âge moyen est de 43 ans. Un tiers a plus de dix ans d'ancienneté dans l'entreprise et un autre tiers moins de deux ans. À travers cette manifestation exceptionnelle par son ampleur, notre intention est de réfléchir sur les formes de socialisation professionnelle qu'elle induit chez les salariés participant aux épreuves sportives. Le fait de participer au Raid renforce-t-il l'appropriation des valeurs de l'entreprise ? Les salariés portent-ils un regard différent sur leur entreprise et sa hiérarchie ? La socialisation particulière vécue durant les épreuves sportives conduit-elle les participants à modifier les relations professionnelles avec leurs collègues de travail ? Le sport peut-il être considéré comme un outil efficace de cohésion interne, voire d'acculturation ?

C'est à l'ensemble de ces questions que nous souhaitons répondre dans cet article. II nous semble que les effets socialisateurs de l'événementiel sportif sur les salariés se mesurent à différents niveaux, au niveau des normes et des valeurs de l'entreprise dont ils facilitent l'appropriation, au niveau de la cohésion interne des groupes en améliorant les relations interpersonnelles et le rapport à la hiérarchie, au niveau enfin de l'attache- ment émotionnel des salariés à l'événement et par ce biais à l'entreprise. Notre article comprend cinq parties. La première vise à rappeler, à travers les différents travaux de recherche, le rôle socialisateur de l'événementiel sportif en entreprise. Les parties sui- vantes reprennent chacun des points évoqués précédemment, à savoir les effets de l'événementiel sportif sur l'appropriation des valeurs, la cohésion interne, le rapport à la hiérarchie et enfin l'attachement émotionnel des salariés à leur entreprise. À la lumière de notre étude de terrain, nous verrons que certains effets socialisants semblent incontestables.

\footnotetext{
${ }^{4}$ Ce que Ramanantsoa (1988, p. 409-410) dénomme l'« identité révélée » en opposition à l'« identité visible » que représente la culture d'entreprise.

${ }^{5}$ L'entreprise ayant souhaité préserver l'anonymat, nous la nommerons par sa fonction générique.
} 


\section{Sport et socialisation professionnelle}

Le Raid développe un concept " sport nature » qui s'inscrit dans la stratégie de l'entreprise. II se déroule sur quatre jours et rassemble entre 1500 et 1800 participants, tous volontaires et salariés du groupe. Ce sont des « collaborateurs et collaboratrices, sans distinction de métier, de nationalité ou de niveau hiérarchique partageant la même joie d'une aventure collective ". Sportifs ou pas, ils sont choisis, à l'issue d'un week-end de sélection, en fonction de leur nombre de participation et leur capacité à représenter les différents secteurs et services de l'entreprise. L'organisation et les moyens mis en œuvre sont très importants. Un train et de nombreux cars sont mis à disposition des participants. Les parcours sont étudiés dans un souci de découverte des richesses de la région. Tous les participants et prestataires sont aussi fortement sensibilisés à la préservation de l'environnement : tri des déchets, gestion des eaux usées, etc. Les produits bio-équitables et biodégradables sont privilégiés. Les équipes (dont le nombre varie entre 160 et 180 représentant une trentaine de pays) se composent de six personnes (4 coureurs et 2 éclaireurs) et sont réparties par catégorie (féminine, masculine, mixte ou vétéran). Elles sont en autonomie complète pendant la journée. Un seul véhicule d'assistance par équipe est autorisé à circuler entre les points d'assistance et à accéder aux parkings assistance. En suivant les instructions de leur road book, les membres de l'équipe se répartissent les tâches en fonction de la nature des épreuves et des consignes données sur des parcours chronométrés. Les épreuves sont diverses et incluent généralement des épreuves d'orientation à pied, des parcours en VTT, du canoë-kayak et des ateliers de cordes. Les éclaireurs assurent la logistique de l'équipe (gestion du matériel, vêtements et ravitaillement) pendant et entre les épreuves sportives, faisant appel à des compétences diverses : sens pratique, organisation, créativité, résolution de problèmes, jeux d'adresse. Un chapiteau géant et de nombreux services (ateliers de massage, réparation du matériel, restauration) sont également mis à disposition des participants. La clôture du Raid est ponctuée par la remise des prix et une soirée festive. Véritable tradition, le Raid est aujourd'hui un événement incontournable dans la vie de l'entreprise.

\section{Le sport comme vecteur de socialisation professionnelle en entreprise}

Les relations entre le sport et l'entreprise sont anciennes. C'est dans les années 1860-1880 que certains entrepreneurs choisissent d'intégrer le sport dans leur politique sociale de ressources humaines (Chartier et Vigarello, 1982 ; Richez et Wahl, 1993 ; Barbusse, 2002). À cette vision paternaliste succède progressivement une vision plus corporatiste. Les premiers championnats apparaissent dans les années 1920 et se multiplient considérablement après la seconde guerre mondiale justifiant la création, en 1952, de l'Amicale des clubs corporatistes. Les compétitions extra-corporatistes s'ouvrent également aux entreprises avec l'apparition d'événements sportifs nationaux comme le Cross du Figaro dans les années 1960 ou le Cross du Républicain lorrain vingt ans plus tard. C'est aussi à cette période que les pratiques sportives en entreprise connaissent un développement important. C'est une période charnière, souligne 
Barbusse, où " l'usage du sport par le monde entrepreneurial se diversifie véritable- ment " (2009, p. 12). Pour Fourré (2003), cela annonce une nouvelle génération de stages sportifs outdoor mais aussi indoor (stages incentive et team building) où les problématiques glissent progressivement vers une finalité plus formative des salariés. La cohésion interne et la synergie des actions sont davantage mises en avant. Des grands groupes tels Apple, Bouygues, SaintGobain, IBM, Microsoft, Nike, GDF Suez, Crédit Immobilier de France ouvrent leurs propres salles de sport dédiées à leurs salariés à qui sont aussi proposés des programmes de coaching individuel et collectif. On voit aussi se multiplier les compétitions intra et inter-entreprises comme par exemple les Business Cup, le Challenge du Lys ou le Challenger's Trophy. Le recours à des personnalités charismatiques issues des sportifs célèbres devient une pratique courante.

Dans le cas du raid organisé par l'entreprise du CAC 40 et étudié dans cet article, le rôle socialisateur de l'événementiel sportif est confirmé par les personnes interviewées. L'analyse de discours nous permet ainsi de clarifier la nature des influences que le Raid exerce sur les salariés.

\section{L'appropriation des valeurs de l'entreprise via le Raid}

Divers travaux visent à mesurer l'impact social des événements sportifs intra et interentreprises en termes de normes et de valeurs. Burlot par exemple (2001) analyse les conséquences sur les salariés de l'implantation d'une salle de remise en forme chez Apple. II souligne notamment que le sport remplit une fonction préventive par l'amélioration de la santé mais aussi une fonction de communication interne et de construction identitaire renforçant le lien entre le salarié et l'entreprise. Ce même auteur étudie quelques années plus tard les conséquences, en termes d'image et d'identification, de la pratique sportive sur les salariés de la société Nil. II note ainsi qu'un « mouvement général d'amélioration de l'image du métier est constaté en fonction du niveau d'engagement dans le club sportif [de l'entreprise] ॥ (2005, p. 85). Ainsi, ceux qui adhèrent au club corporatiste ont une perception plus positive de leur entreprise (ils la jugent " juste et humanisée ») et adhèrent davantage aux valeurs de l'entreprise que ceux qui ne sont pas adhérents. Fourré (2007) de son côté montre comment la participation des salariés à des événements et des stages proposés en interne est fortement encouragée par la direction. Pour cet auteur, le recours aux rituels, à la mobilisation des symboles et des codes mêlant la dynamique sportive et l'image de l'entreprise permettent d'élever le niveau d'adhésion du personnel aux normes et aux valeurs de l'entreprise.

À l'échelle de l'entreprise du CAC 40 que nous avons étudiée, il apparaît nettement que l'événement sportif est un relais favorisant l'appropriation par les salariés des valeurs du groupe. L'événement sportif en question est un raid qui vient de fêter ses 20 ans d'existence. Parmi les quatre catégories de valeurs identifiées par Tribou (2011), ce raid entre dans la catégorie des valeurs prônant la " fraternité sportive ${ }^{6}$ " en mettant en avant l'esprit du collectif

\footnotetext{
${ }^{6}$ Les trois autres valeurs proposées par l'auteur sont : le dépassement de soi (valeur compétitive), la juste compétition (valeur attractive) et l'amateurisme (valeur humaniste).
} 
et la cohésion de groupe dans une ambiance festive et ludique ${ }^{7}$. Cet événement sportif est aujourd'hui majeur dans la culture de l'entreprise et constitue une tradition incontournable au sein du groupe. Le Raid, peut-on lire sur le site de l'entre- prise, " c'est quatre jours durant lesquels s'expriment pleinement, intensément les valeurs essentielles et constitutives de notre histoire, de notre identité et de nos métiers. II symbolise chaque année et de belle manière l'esprit de groupe ". II ne fait guère de doute que les valeurs affichées durant le Raid sont celles de l'entreprise qui trouve dans ces épreuves un formidable support de communication interne. Nos interviewés confirment cette proximité de valeurs. "Le Raid traduit bien les valeurs du groupe (explique Pierre, cadre, chargé de communication, première participation au Raid). Par ce biais, on les éprouve et on se les approprie plus facilement. " Pour Alexandre (cadre, chargé de communication dans un service de la direction générale, quatre participations à son actif), " Les valeurs de l'entreprise : exigence, audace, engagement et cohésion, on les retrouve facilement pendant les quatre jours. C'est aussi l'occasion de découvrir la richesse et la diversité du groupe et de ses nombreuses filiales. "Les valeurs de l'entre- prise, souligne Guillaume (responsable commercial, cadre, quatrième participation), " c'est exactement celles que doivent intégrer les raiders ". Grâce au Raid, ajoute Jean- Michel (délégué syndical, première participation), « il se crée une grande solidarité que je ne connaissais pas auparavant et qui se poursuit après ". Cet esprit de groupe est largement mis en avant par l'entreprise. C'est aussi l'occasion de sensibiliser les salariés sur la préservation de l'environnement et le développement durable, thèmes phares dans la politique du groupe.

\section{Un effet important sur la cohésion interne}

Si le recours à l'événementiel sportif à des fins de management et de communication interne a été souligné à maintes reprises (Cegarra, 1990 ; Jallat et Mestressat, 1995 ; Burlot et Pichot, 2004 ; Pierre, 2009), c'est en partie pour démontrer sa capacité à créer de la cohésion interne. " En tant qu'instrument de mobilisation des ressources humaines, le sport relève essentiellement d'une stratégie de management des RH », souligne Barbusse (2009, p. 12). Étudiant le Défimmo, raid sportif lancé en 1995 par le Crédit immobilier de France (CIF), Burlot et Tribou (2004) insistent sur le sentiment d'appartenance qui sort renforcé par le vécu commun de salariés de statuts hiérarchiques différents mais partageant une même expérience sportive et humaine. Dans leur enquête sur diverses entreprises dont Apple et 3M, Pierre, Pichot et Burlot (2010) insistent sur l'effet de "culture réseau " que le sport autorise en multipliant les interfaces entre les salariés. Dans notre étude, la plupart des salariés ayant participé au raid reconnaissent que leurs relations avec leurs collègues ont évolué avec l'événement. Tous louent son effet positif sur la cohésion du groupe durant l'épreuve et après. " J'ai fait des connaissances, nous dit Johan (chargé de projet au service communication, première participation), et cela n'a pas de prix. " "J'ai rencontré des personnes formidables d'horizons différents, de métiers différents et surtout plein de profils qui se complètent les uns

\footnotetext{
7 Notons avec Pierre et Tribou (2013) que les entreprises font davantage le choix aujourd'hui de pratiques sportives ludiques et festives plutôt que compétitives et poussant au dépassement de soi (pra- tiques très en vogue dans les années 1980).
} 
aux autres "(Pierre). Le raid permet aux salariés de se retrouver autour d'un même " dénominateur commun " qui dépasse les frontières, les enjeux et les hiérarchies (Alexandre). Le raid, ajoute notre interlocuteur aguerri à cet événement, "c'est le Graal de la com' interne ". Pour autant, cette cohésion interne ne perdure pas nécessairement au-delà de l'événement, ou en tout cas, elle reste surtout confinée aux groupes sportifs constitués. Si le raid, comme le reconnaît Jean-François (cadre gestionnaire à la direction des services partagés et dont c'est la troisième participation), " recrée le contexte d'entreprise mais avec des liens beaucoup plus forts ", la proximité relationnelle qu'il favorise concerne essentiellement les anciens équipiers du raid. Dès lors que ces derniers se retrouvent éparpillés dans leurs différents services d'affectation, il est difficile d'affirmer que leur complicité bénéficie durablement à l'entreprise. C'est ce que nous explique Charles (salarié au centre des recherches, première participation) : " Je ne suis pas plus en adéquation avec mon entreprise. II n'y a pas de côté affectif entre moi et mon entreprise. Durant le raid, tu rencontres des gens et c'est avec ces personnes-là que tu mets en place ce côté affectif mais pas avec la boîte. "Cela étant, il semble indéniable que le raid contribue à générer une convivialité entre les individus dont l'entreprise, dans sa politique interne, sait tirer bénéfice par le recours massif à des blogs, vidéos, images, et autres informations postées régulièrement sur ses sites. " On ne retrouve ce niveau de cohésion dans aucun autre événement interne ", explique Florence (salariée à la direction des services partagés, deuxième participation). " On aborde les travaux de groupe plus facilement. C'est plus facile depuis le Raid ", souligne Anaïs (chargée de missions $\mathrm{RH}$, cadre à la direction générale, première participation).

\section{Le raid comme facteur atténuateur de la hiérarchie}

Si de nombreux auteurs soulignent le rôle mobilisateur du sport, son effet sur la cohésion interne, son utilité en tant que relais des valeurs de l'entreprise, peu tentent de mesurer l'impact au niveau hiérarchique. Dans un article de 2006, Pierre décortique le discours des salariés d'Adidas et montre comment l'incitation au sport au sein de l'entreprise est perçue différemment par ces derniers, soit comme une valeur altruiste optimisant un rapport gagnant/gagnant entre les salariés et leur hiérarchie, soit au contraire comme un " don coercitif " qui, en compensation d'un " culte du privilège ${ }^{8}$ ", imposerait une obligation de performance chez les salariés (2006, p. 77). Pierre et Tribou relèvent ces cas de résistance et de refus venant de salariés méfiants qui relativisent le caractère désintéressé de ces initiatives émanant de leur hiérarchie. II est vrai qu'en certaines circonstances, l'événementiel sportif est parfois utilisé comme un outil " révélateur de personnalité " (Pierre et Tribou, 2013, p.124) permettant à la direction d'observer le comportement social de ses salariés soumis à un environnement insolite et générateur de stress et de tensions (voir également les travaux de Picq à ce propos). Le risque d'instrumentalisation n'est jamais loin. Pour autant, une des conséquences de l'événementiel

\footnotetext{
${ }^{8}$ À travers les notions de " don coercitif " et de "culte du privilège ", l'auteur veut signifier que les salariés se sentent redevables envers l'entreprise qui leur accorde des privilèges. Ils ont une " dette " envers elle et doivent se " montrer à la hauteur " pour justifier les avantages que leur hiérarchie consent à leur octroyer.
} 
sportif interne nous paraît être de favoriser une réelle " atténuation des frontières hiérarchiques " (Pierre et Tribou, op. cit.). S'appuyant sur leur étude au sein du CIF, Burlot et Pichot parlent même d' "écrasement d'une logique hiérarchique ou d'une logique de rang professionnel »(2004, p. 235). Le sport est un moyen de réduire les distances hiérarchiques, précisent les auteurs, par la simplicité et la proximité des contacts humains que l'événement ad hoc rend francs et directs. L'environnement spécifique qu'il autorise amène les joueurs, quel que soit leur grade hiérarchique à " mouiller ensemble le maillot ", à porter une tenue uniforme et parfois même à inverser les rapports de force entre cadres et employés (Fourré, 2007). En s'affranchissant de l'ordre hiérarchique régissant les relations professionnelles, ces situations particulières, ajoute Pichot, " atténuent la force de la relation contractuelle du salarié à son entreprise " (2007, p. 241). Dans le réseau informel des sportifs, le rang hiérarchique est ainsi gommé au bénéfice des seules qualités individuelles et collectives qui font la force du groupe et de l'équipe (Pierre et al. 2010). Nos entretiens confortent les conclusions des auteurs. Pour Morgane (salariée du service logistique, deuxième participation), le raid a cette spécificité de mettre « tout le monde sur un pied d'égalité, que l'on soit cadre ou pas, nous sommes tous avec les mêmes compositions d'équipes et sur les mêmes épreuves ". Pour Hugues (délégué syndical) dont la participation au Raid est très récente, " il y a un grand aspect fédérateur, sans notion de hiérarchie, et la structure de l'entre- prise est mise de côté au profit d'un aspect bon enfant, agréable et très sincère ". Notons que ceux qui se montrent les plus réceptifs aux effets atténuateurs des rapports hiérarchiques sont souvent les salariés et représentants syndicaux. Pour Arnaud (président syndical, deuxième participation), " c'est un moment très convivial, nous pouvons discuter avec le PDG car il est très accessible. II nous rend visite le dernier jour et participe à la remise des prix. Il est avec ses salariés. J'ai même eu la chance de pouvoir discuter avec lui et d'être pris en photo en sa présence ". Aux dires de la plupart de nos inter- locuteurs, cette proximité nouvelle facilitée par l'épreuve sportive est une aubaine qui rend le management plus humain et accessible.

\section{L'attachement émotionnel à l'entreprise}

Dans leur politique de ressources humaines, l'objectif des employeurs, note Barbusse (2002, p. 409) n'est pas seulement d'améliorer la santé de leurs salariés, gage d'une meilleure performance. Il est aussi de favoriser chez ces derniers une adhésion institutionnelle et affective. Le salarié ne doit pas être seulement lié à son entreprise par le contrat de travail (vision tayloriste), il doit être également lié par un sentiment communautaire d'appartenance. En cela, le sport joue un rôle moteur très important. Pierre et Tribou évoquent cet attachement émotionnel lié à l'événementiel sportif : "L'exposition à un événement sportif est susceptible de générer un état d'excitation mobilisateur et par conséquent de provoquer un attachement émotionnel des publics, tant externes qu'internes à l'entreprise qui est associée » (2013, p. 121). Ces aventures collectives, note Pichot, contribuent ainsi à renforcer les « transferts affectifs »(2007, p. 234) entre l'événementiel sportif et l'entreprise organisatrice. Ainsi, il semble établi, par les différents auteurs cités, que la gestion et la mobilisation des salariés autour d'un événement sportif génèrent un attachement informel à l'entreprise qui séduit de 
plus en plus les dirigeants. Dans le cas du raid que nous avons étudié, cet attachement est d'autant plus marqué que la sélection des candidats au raid est basée sur le volontariat (la priorité est toutefois donnée à ceux n'y ayant encore jamais participé). Nos interviews montrent que les motivations des participants sont multiples : les raideurs souhaitent créer des liens avec leurs collègues venus des autres régions de France et de l'étranger (Australie, Italie, Espagne). Ils veulent aussi participer à un événement qu'ils savent extraordinaire par son ampleur, mêlant un réel esprit d'équipe et une entraide mutuelle. Le raid est aussi, pour eux, un moyen de se mesurer aux autres et à eux-mêmes et de repousser leurs limites. L'attachement affectif au raid et par voie de conséquence à l'entreprise est évoqué par nos interlocuteurs : " J'ai eu le sentiment d'appartenir à une grand famille " explique Hugues. Cette expérience, précise-t-il, permet de voir différemment les choses. "II y a incontestablement un côté affectif qui s'est développé grâce au Raid, raconte Pascal (costofficer, première participation), particulièrement sur le plan humain. J'ai gardé un très bon relationnel avec l'ensemble des participants. Affectif car lors de nos derniers échanges avec ces personnes, le dialogue était chaud en émotion, partage, valeurs communes, toutes ces choses qu'on a pu partager lors du Raid et qu'on réévoque au travail. "Beaucoup de nos interviewés insistent sur la dimension émotionnelle de l'événement. La présence de la direction et sa grande accessibilité pendant les épreuves et lors de la remise des récompenses est une réelle occasion de renforcer les liens et l'adhésion des salariés à leur entreprise. II y a surtout, explique ce président syndical cité plus haut, " une notion d'affectivité avec sa direction dont on défend les couleurs qui est très importante ". Grâce au Raid, souligne Anaïs cadre à la direction générale, " on a le sentiment d'exister et d'être mieux considéré ". " Du coup, ajoute Sandrine (salariée du service $\mathrm{RH}$, première participation) notre regard sur l'entreprise et ceux qui la dirigent est plus positif et constructif. »

\section{Conclusion}

Parmi les possibles usages du sport en entreprise, nous avons souhaité insister sur ceux qui influent sur la socialisation des salariés. Dans une perspective de politique de communication interne, le sport est à l'évidence un « produit communicationnel pour les entreprises en même temps qu'une ressource pour les salariés " (Pichot, 2007, p. 232). Certes, nous l'avons évoqué plus haut, cela n'exclut nullement la tentation de l'instrumentalisation, le sport s'inscrivant alors dans une recherche de performance éco- nomique et managériale. Mais ne retenir que cette dimension apparait réducteur. L'évènementiel sportif est aussi un moyen pour les entreprises de densifier les relations sociales avec leurs salariés et de créer des conditions favorables pour une plus grande cohésion interne et un rapprochement institutionnel pérenne. La logique interne du Raid organisé par l'entreprise du CAC 40 entre dans cette perspective. Son impact sur les salariés est réel. L'appropriation des valeurs de l'entreprise est amplifiée chez ces derniers (on observe d'ailleurs que cela concerne souvent les cadres dont la position hiérarchique suppose un minimum d'adhésion à la culture de l'entreprise) à plus forte raison si ils les vivent et les éprouvent dans l'intensité de l'effort sportif et collectif. Les relations particulières que l'événement génère créent des liens forts qui perdurent. Même s'ils restent confinés aux anciens équipiers du raid, ils facilitent la communication interne entre les services concernés 
dont l'effet sert utilement l'entreprise. Les salariés, en particulier ceux évoluant dans les services à fortes relations humaines, confirment l'impact positif du raid sur la cohésion interne et le renforcement des liens entre les collaborateurs du groupe. Les salariés et représentants syndicaux apprécient le Raid pour son effet atténuateur des rapports hiérarchiques. Pour la plupart de nos interviewés dont la participation, il est vrai, reste un acte volontaire, l'attachement affectif au raid est perceptible. Tous parlent de leur participation et de ce qu'ils ont partagé avec émotion et plaisir. Si la position objective des participants dans l'entreprise semble agir sur la nature du rapport subjectif qu'ils entretiennent avec le raid, on peut observer égale- ment une corrélation entre le nombre de participations de nos interviewés et l'enthousiasme et le regard positif qu'ils portent sur le raid et ses incidences sur l'entreprise. Les éditions successives de l'événement permettent d'entretenir les souvenirs et de raviver la mémoire collective. Le vécu et l'expérience humaine partagés par les participants au Raid restent durablement ancrés et influent, d'une manière certes plus ou moins appuyée selon les individus, sur la socialisation interne des salariés. L'image qu'ils portent sur leur entreprise en sort améliorée. Le rapport à la hiérarchie et la perception des dirigeants sont facilités. Le raid, " c'est un moment unique et privilégié ", souligne Arnaud. Ce sont des relations fortes " qui n'ont pas de prix ", explique Thierry (cadre et directeur de projet à la direction des équipements, première participation). C'est " un niveau d'émulation qu'on ne retrouve pas ailleurs " (Alexandre). "C'est plus facile depuis le raid. On aborde les problèmes différemment et solidairement. On s'entraide même au travail ", souligne Johan, reconnaissant que cela l'a conduit à un plus fort investissement professionnel depuis. Pour Amélie (salariée du service logistique, première participation), " il y a un avant et un après ", les relations au sein du groupe sont meilleures, amenant les salariés à se sentir affectivement et culturellement plus proches de leur entreprise. Grâce au raid, c'est ainsi tout un processus de socialisation interne qui agit et souligne le rôle intégrateur et rassembleur de l'événement sportif dans l'entreprise.

dbernardeaumoreau@free.fr marie.hingant@u-pem.fr 


\section{Références bibliographiques}

BARBUSSE B. (1997), Sport et entreprise : des apports réciproques en matière de gestion des ressources humaines, thèse de doctorat non publiée, Université de Paris-5.

BARBUSSE B. (2002), "Sport et entreprise : des logiques convergentes ? ", L'Année sociologique, 2, 52, p. 391-415.

BARBUSSE B. (2009), "Entre sport et entreprise, une attirance réciproque ", L'Expansion Management Review, 3, 134, p. 10-19.

BERNARDEAU-MOREAU D. (2004), Sociologie des fédérations sportives : la professionnalisation des dirigeants bénévoles, Paris, L'Harmattan.

BURLOT F. (2001), " L'intégration du sport dans une politique de gestion des ressources humaines. Le cas d'Apple ", dans M. Desbordes, Stratégies des entreprises dans le sport. Acteurs et management, Paris, Economica, p. 181-195

BURLOT F. (2005), " Sport et identité d'entreprise. Vers une modification de l'imaginaire organisationnel », Staps, 3, 69, p. 73-92.

BURLOT F., PICHOT L. (2004), " L'événement sportif et la cohésion des salariés en entreprise ", dans M. Desbordes, Stratégies des entreprises dans le sport, Paris, Economica, p. 221-248.

CEGARRA J.-J. (1990), « Sponsorisme et mécénat », Direction et gestion, 124-125, p. 73-80.

CHARTIER R., VIGARELLO G. (1982), "Les trajectoires du sport. Pratiques et spec- tacle ", Le Débat, 19, p. 35-58.

DUBAR C. (2000), La Socialisation : construction des identités sociales et professionnelles, Paris, A. Colin.

EHRENBERG A. (1991), Le Culte de la performance, Paris, Calmann-Lévy. FOURRÉ C. (2000), Mise en scène de la métaphore sportive dans la formation professionnelle : l'exemple des stages outdoor organisés pour les cadres d'entreprise, thèse de doctorat non publiée, laboratoire de recherche en APS de I'UFRAPS, Université de Toulouse-3.

FOURRÉ C. (2003), « Le secteur des stages outdoor: la professionnalisation des consultants d'entreprises ", Revue européenne de management du sport, 9, p. 35-72.

FOURRÉ C. (2007), « Mise en scène de la métaphore sportive dans la formation professionnelle des cadres d'entreprise : l'exemple des stages outdoor ", communication au colloque "Sport et 
Travail », Lille, 10-12 décembre.

JALLAT F., MESTRESSAT A. (1995), " De la communication événementielle au sein de l'entreprise: le mécénat comme vecteur privilégié de motivation interne ", Revue française du marketing, 151, p. 53-62.

LEFEVRE N. (2007), Le Cyclisme d'élite français : un modèle singulier de formation et d'emploi, thèse, Université de Nantes.

LE GOFF J.-P. (1992), Le Mythe de l'entreprise, critique de l'idéologie managériale, Paris, La Découverte.

LOIRAND G. (2003), " Les paradoxes de la professionnalisation des associations sportives ", dans L. Prouteau (dir.), Les Associations entre bénévolat et logiques d'entreprise, Rennes, Presses universitaires de Rennes, p. 85-103.

MEIGNANT A., RAYER J. (1991), Saute manager, Paris, Les Éditions d'organisation.

PICHOT L. (2007), « Les politiques de communication par le sport au service de la dynamique organisationnelle des entreprises ", Communication et organisation [en ligne], 31 | 2007, http://communicationorganisation.revues.org/222.

PICQ T. (2005), « Comment développer la performance collective ? Quand le handballeur vient au secours du manager ", Gérer et comprendre, 79 (3), p. 76-83.

PIERRE J. (2006), " Le recours au sport chez Adidas. Effets des discours et des pratiques managériales sur l'implication des cadres », STAPS, 74 (2), p. 69-84.

PIERRE J. (2009), " S'investir corps et âme en entreprise. Contribution à une sociologie de la mobilisation des cadres par le sport ", thèse de doctorat en STAPS, Université de Strasbourg.

JULIEN P., PICHOT L., BURLOT F. (2010), " Le sport en entreprise au service des pratiques managériales », Communication, 28 (1), p. 133-160.

PIERRE J., BARTH I. (2013), « Sport et management : un dialogue ancien, de nouvelles perspectives ", RIMHE, Revue interdisciplinaire management, homme(s) \& entreprise, 2 (6), p. 2-4.

PIERRE J., TRIBOU G. (2013), « L'événementiel sportif comme outil de communication interne en entreprise ", Gestion 2000, 30 (3), p. 113-128.

RAMANANTSOA B. (1988), "L'identité ", dans J.-P. Anastasopoulos (dir.), Strategor, Paris, 
InterÉdition, p. 401-472.

RICHEZ J.-C., WAHL A. (1993), La Vie quotidienne en Alsace. Entre France et Allemagne 18501950, Paris, Hachette.

SEKIOU L., BLONDIN L., BESSEYRE DES HORTS C.-H., CHEVALIER F. (1993), Gestion des ressources humaines, Paris, De Boeck Université.

TRIBOU G. (2011), Sponsoring sportif, $4^{\text {ème }}$ édition, Paris, Economica. 